\title{
A doação voluntária de sangue sob a ótica de universitários da área da saúde
}

\author{
Voluntary blood donation from the perspective of university students in the health field \\ La donación voluntaria de sangre desde la perspectiva de los estudiantes universitarios del campo \\ de la salud
}

Recebido: 09/04/2021 | Revisado: 16/04/2021 | Aceito: 19/04/2021 | Publicado: 04/05/2021

\author{
Teodoro Marcelino da Silva \\ ORCID: https://orcid.org/0000-0002-5817-4244 \\ Universidade Regional do Cariri, Brasil \\ E-mail: teodoro.marcelino.s@gmail.com \\ Ingrid Mikaela Moreira de Oliveira \\ ORCID: https://orcid.org/0000-0002-8901-362X \\ Universidade Estadual do Ceará, Brasil \\ E-mail: ingrid_lattes@hotmail.com \\ Natália Bastos Ferreira Tavares \\ ORCID: https://orcid.org/0000-0002-1139-600X \\ Universidade Regional do Cariri, Brasil \\ E-mail: nataliabastosf@hotmail.com
}

\begin{abstract}
Resumo
O estudo teve como objetivo identificar a percepção de universitários da área da saúde sobre a atitude da doação voluntária de sangue. A natureza do estudo é exploratória-descritiva, com abordagem qualitativa, desenvolvido, no ano de 2020, com os estudantes da área da saúde de uma universidade pública cearense. Os dados foram obtidos mediante um questionário virtual e interpretados conforme a técnica de análise categorial. Os resultados foram apresentados por meio de duas categorias oriundas da análise de conteúdo, a saber: 1. Doação de sangue como ato de solidariedade e de amor que salva vidas; 2. Necessidade da doação de sangue em situações envolvendo acidentes, doenças e cirurgias. Posteriormente foram discutidas mediante a literatura científica pertinente à temática em estudo. Conforme os resultados encontrados, pôde-se concluir que apesar da maioria dos acadêmicos não serem doadores de sangue, eles reconhecem a doação de sangue como uma atitude voluntária, solidária e altruísta que tem a capacidade de salvar vidas, e sendo amplamente utilizada em procedimentos médicos, doenças e acidentes, a qual, normalmente, faz-se necessária em transfusões sanguíneas.
\end{abstract}

Palavras-chave: Doação de Sangue; Doador voluntário; Estudantes de ciências da saúde; Solidariedade.

\begin{abstract}
The study aimed to identify the perception of university students in the health field about the attitude of voluntary blood donation. The nature of the study is exploratory-descriptive, with a qualitative approach, developed in the year 2020 , with students in the health area of a public university in Ceará. The data were obtained through a virtual questionnaire and interpreted according to the categorical analysis technique. The results were presented through two categories derived from content analysis, namely: 1 . Blood donation as an act of solidarity and love that saves lives; 2 . Need for blood donation in situations involving accidents, illnesses and surgeries. Subsequently, they were discussed using the scientific literature relevant to the subject under study. According to the results found, it was possible to conclude that although most academics are not blood donors, they recognize blood donation as a voluntary, supportive and altruistic attitude that has the ability to save lives, and is widely used in medical procedures, illnesses and accidents, which is usually necessary in blood transfusions.
\end{abstract}

Keywords: Blood donation; Voluntary donor; Health sciences students; Solidarity.

\section{Resumen}

El estudio tuvo como objetivo identificar la percepción de los estudiantes universitarios del campo de la salud sobre la actitud de la donación voluntaria de sangre. La naturaleza del estudio es exploratorio-descriptivo, con enfoque cualitativo, desarrollado en 2020, con estudiantes del área de salud de una universidad pública de Ceará. Los datos se obtuvieron a través de un cuestionario virtual y se interpretaron según la técnica de análisis categórico. Los resultados se presentaron a través de dos categorías derivadas del análisis de contenido, a saber: 1. La donación de sangre como acto de solidaridad y amor que salva vidas; 2 . Necesidad de donar sangre en situaciones de accidentes, enfermedades y cirugías. Posteriormente, se discutieron con base en la literatura científica relevante al tema en estudio. De acuerdo con los resultados encontrados, se puede concluir que aunque la mayoría de los académicos no son donantes de sangre, reconocen la donación de sangre como una actitud voluntaria, solidaria y altruista que tiene la capacidad de 
salvar vidas y es muy utilizada en procedimientos médicos, enfermedades y acidentes, que suele ser necesario en las transfusiones de sangre.

Palabras clave: La donación de sangre; Donante voluntario; Estudiantes de ciencias de la salud; Solidaridad.

\section{Introdução}

O processo de doação voluntária de sangue, até os dias atuais, ainda se caracteriza como uma questão de interesse mundial, o que se justifica mediante a inexistência de uma substância que possa substituir o tecido sanguíneo artificialmente (Carlesso et al., 2017). Constitui-se em um gesto reconhecido internacionalmente como solidário, onde o doador permite a retirada de uma certa quantidade do seu próprio sangue, para que possa ser armazendo em locais específicos (banco de sangue e/ou hemocentro) e assim, ser utilizado em uma transfusão sanguínea (Malheiros, Oliveira, Pinheiro, Monteiro \& Abreu, 2014).

Desde eventos como o surgimento do Programa Nacional do Sangue (PRÓ-SANGUE), em 1980; bem como a criação do Plano Nacional de Sangue e Hemoderivados (PLANASHE), em 1988 e a Lei 7.649/1988, dentre várias reivindicações ocorridas na época, visando a regulamentação dos serviços de hemoterapia e que teve como pauta a garantia de sangue seguro para toda população; o ato de doar sangue passou a ser considerado um ato voluntário e humanitário, sendo proibida a livre comercialização de sangue, além da obrigatoriedade da realização dos cadastros dos doadores e a realização de exames laboratoriais do sangue doado, objetivando a segurança dos receptores (Brasil, 2015).

Nesse sentido, com o decorrer dos anos, verifica-se que a necessidade de transfusões de sangue tem aumentado de forma constante, ao passo que ocorrem com muita frequência, acidentes, violências, intercorrências obstétricas e puerperais, morbidades, além da sua utilização em procedimentos médicos de maior complexidade, como é o caso de cirurgias, especialmente as cirurgias cardíacas e transplantes (Amorim \& Baldessar, 2019). Neste contexto que é a realidade de muitos países, incluindo o Brasil, é preocupante, pois as doações espontâneas de sangue não suprem as demandas por transfusões sanguíneas que habitualmente são emergenciais (Loks et al., 2019).

No Brasil, infelizmente, o percentual de doadores voluntários ainda se encontra abaixo do ideal, já que a Organização Mundial de Saúde (OMS) propõe que o percentual ideal estaria entre 3 a $5 \%$ da população, sendo que no cenário brasileiro apenas 1,8\% são doadores fidelizados (Pereira et al., 2016). Desse modo, recrutar candidatos elegíveis ou fidelizar os que já doaram alguma vez, torna-se cada vez mais necessário para a manutenção dos bancos de sangue e/ou hemocentros, para que assim possa ter bolsas de sangue suficientes para garantir as demandas de transfusões sanguíneas (Otéro, Marques, Santos, González \& Iglesia, 2020). Assim, torna-se relevante o desenvolvimento de estratégias com vista à sensibilização da população (Nascimento, Ilha, Marzari, Diefenbach \& Backes, 2015).

Diante desta problemática, várias pesquisas ressaltam a necessidade de desenvolver métodos que visem recrutar estudantes das instituições do ensino superior, uma vez que o público jovem, habitualmente, é considerado como potenciais doadores de sangue por apresentarem um bom estado de saúde e muitos anos de vida pela frente para realizarem doações. Destarte, estudos apontam a importância de se conhecer a percepção dos universitários sobre o processo de doação voluntária de sangue (Otéro et al., 2020; Bousquet, Aleluia \& Luz, 2018; Raghuwanshi, Pehlajani \& Sinha, 2016), principalmente, dos estudantes de graduação e profissionais da área da saúde, visto que normalmente reconhecem a importância do tecido sanguíneo para os serviços de saúde. Logo, espera-se que os estudantes e/ou profissionais de saúde orientados sobre a importância da doação de sangue, sejam doadores habituais e constantemente estimulem a população em geral a realizar este gesto solidário e vital (Amorim \& Baldessar, 2019)

A escolha em investigar o processo de doação voluntária de sangue sob à ótica dos universitários da área da saúde, justifica-se mediante a realização de uma revisão de literatura prévia onde evidenciou a escassez de estudos sobre a temática e quantidade expressiva de estudantes que não são doadores de sangue, em virtude de inaptidão clínica e desconhecimento sobre 
o processo de doação. Dito isso, este estudo se torna relevante por proporcionar visibilidade à temática e assim fornecer subsídios para o desenvolvimento de estratégias de captação voltadas a esses graduandos, em especial aos da área da saúde, com vista a sensibilizar a se tornarem doadores voluntários e garantirem a quantidade necessária de bolsas de sangue para suprir as transfusões diárias. Neste intento, destaca-se a seguinte questão da pesquisa: qual é a percepção dos universitários da área da saúde sobre a atitude da doação voluntária de sangue? Tendo em vista os aspectos apresentados, este estudo teve como objetivo identificar a percepção de universitários da área da saúde sobre a atitude da doação voluntária de sangue.

\section{Método}

Trata-se de um estudo exploratório-descritivo, com abordagem qualitativa, cuja elaboração atendeu às recomendações dos Critérios Consolidados para Relato de Estudos Qualitativos (COREQ) (Minayo, 2017). Os participantes da pesquisa foram os estudantes universitários dos cursos de Educação Física e Enfermagem, que são os únicos cursos de graduação da área da saúde de uma universidade pública da região centro-sul cearense, localizada na zona urbana do município de Iguatu-CE.

Foram incluídos, os acadêmicos regularmente matriculados do primeiro ao décimo semestre no curso de graduação em Enfermagem e do primeiro ao oitavo, no curso de Educação Física, no semestre letivo 2020.1, os quais dispunham de equipamentos tecnológicos com acesso à internet, para que pudessem responder o instrumento de coleta de dados. Desse modo, adotou-se como critério de exclusão, estudantes que por motivos de doença e/ou licença maternidade estavam afastados das atividades acadêmicas.

Os participantes da pesquisa foram selecionados mediante o uso da técnica metodológica snowball, amplamente conhecida como Snowball Sampling. Técnica, esta, traduzida no Brasil como "Bola de Neve" ou "Amostragem em Bola de Neve". A técnica snowball, caracteriza-se por ser um método de amostragem não-probabilística, amplamente utilizada em estudos voltados às ciências sociais, a qual os participantes iniciais indicam outros participantes com as mesmas características esperadas no estudo, e estes indicam novamente outros participantes e assim sucessivamente. Dessa forma, o quadro de amostragem pode crescer a cada entrevista, caso seja do interesse do pesquisador (Baldin \& Munhoz, 2011; Vinuto, 2014).

A pesquisa foi realizada durante os meses de março a dezembro de 2020. Sendo que em decorrência do surgimento do novo coronavírus, denominado internacionalmente como SARS-CoV-2 (Severe Acute Respiratory Syndrome Coronavirus 2), responsável por causar a síndrome gripal e/ou síndrome respiratória aguda grave em humanos (Coronavírus Disease-2019 ou COVID-19), a qual impactou negativamente no setor educacional, culminando no período de quarentena com o isolamento e distanciamento social, o que fez com que este estudo fosse desenvolvido de forma remota (on-line).

Para alcançar os objetivos do estudo, utilizou-se como instrumento de coleta de dados, um questionário virtual elaborado através da ferramenta Google forms $®$, contendo os dados de caracterização sociodemográfica, afetivo sexual e sanguíneo dos participantes, seguido por um roteiro de perguntas previamente estruturado: Você já doou sangue voluntariamente? Caso já tenha sido um doador de sangue, qual a principal razão que o fez tomar essa atitude? Para você, qual a importância da doação de sangue? Você reconhece a doação de sangue como um ato de importância social? Por quê? O questionário foi disponibilizado para preenchimento através da rede social WhastsAPP®.

As pesquisas realizadas no ambiente virtual, por intermédio dos instrumentos de coleta de dados on-line, tais como os questionários, demonstraram ser uma estratégia essencial e corriqueira para coleta de dados, durante o cenário pandêmico de COVID-19. Desse modo, os questionários virtuais proporcionam melhorias e uma rapidez na obtenção dos dados pretendidos no estudo, além de oportunizar ao (s) pesquisador (es) um contato rápido e preciso com os participantes da pesquisa (Faleiros et al., 2016).

A coleta de dados foi encerrada mediante o critério de saturação teórica, que é quando os depoimentos suprem os dados esperados para conclusão das ideias do estudo, sem que haja a necessidade de persistir na coleta de novos dados 
(Nascimento et al., 2018). Posteriormente, realizou-se as transcrições das respostas fornecidas pelos participantes do estudo. Os dados obtidos foram tratados conforme a técnica de análise de conteúdo, especificamente utilizando-se da modalidade de Análise Categorial Temática (Bardin, 2016), cumprindo-se as três principais etapas desse processo: a pré-análise; a exploração do material; e o tratamento dos resultados, inferência e a interpretação.

Após o processo analítico dos dados, emergiram duas categorias: 1) Doação de sangue como ato de solidariedade e de amor que salva vidas; 2) Necessidade da doação de sangue em situações envolvendo acidentes, doenças e cirurgias. Com vista a atender o anonimato da pesquisa, os acadêmicos foram identificados mediante a letra $\mathrm{E}$, referindo-se à palavra estudante, seguido de um numeral ordinal que correspondeu a ordem a qual os acadêmicos responderam (via on-line) o instrumento de coleta de dados, exemplo: E1, E2, E3, sendo assim, assegurado a confidencialidade e o sigilo das informações fornecidas pelos participantes do estudo.

A pesquisa se deu em conformidade com os princípios éticos e legais da Resolução 466/12 do Conselho Nacional de Saúde do Ministério da Saúde, respeitando todas as recomendações que envolvem pesquisas com os seres humanos. Essa pesquisa foi inserida na Plataforma Brasil e avaliada pelo Comitê de Ética em Pesquisa, obtendo parecer favorável consubstanciado de $\mathrm{n}^{\circ} 4.270 .207 / 2020$. Todos os participantes foram informados e orientados sobre os aspectos pertinentes ao desenvolvimento do estudo, seus objetivos, os resultados esperados e acerca da sua participação voluntária, por meio do Termo de Consentimento Livre e Esclarecido (TCLE). Ressalta-se que todos os participantes concordaram e assinaram virtualmente o referido termo.

\section{Resultados e Discussão}

Os dados de caracterização dos participantes apontaram que a pesquisa foi constituída por 27 estudantes, 15 do curso de graduação em Enfermagem e 12 do curso de Educação Física, com predomínio dos matriculados regularmente no $7^{\circ}$ semestre, de ambos os cursos. A faixa etária dos participantes variou de 17 a 40 anos, com média de 25,4 anos, prevalecendo estudantes do sexo feminino. A grande maioria se autodeclarou pardos, solteiros, heterossexuais, sem vínculo empregatício, católicos, com renda familiar bruta de um a dois salários mínimos (considerando o valor base de $\mathrm{R} \$ 1.045,00$ vigente durante o período da coleta de dados) e pertencentes ao grupo sanguíneo 0+. Da análise das falas, emergiram as duas categorias, a seguir:

\section{Categoria 1: Doação de sangue como ato de solidariedade e de amor que salva vidas.}

Esta categoria reflete a percepção dos acadêmicos sobre a doação de sangue, considerando-a como um ato solidário e de amor que possibilita salvar vidas, também compreendida como uma atitude voluntária e simples, contudo valiosa para a sobrevivência humana, cujo objetivo é ajudar quem estiver necessitando, como fica evidenciado nos discursos abaixo:

"Forma de ajudar o próximo" (E09). "[...] Ato solidário e vital que pode salvar vidas daqueles que necessitam [...]" (E13). "É um ato de amor e solidariedade que salvam vidas [...]" (E15). "Ato solidário, ajuda a salvar outras vidas" (E18). "[...]é um ato de amor ao próximo, já que muitas das vezes o doador não sabe para quem está doando" (E20). "É um ato de solidariedade. Ajudar pessoas que necessitam" (E21). "É muito importante pois salva vidas" (E23). "É algo que se torna muito valioso por aquele simples ato de poder salvar uma vida" (E25). "É um ato solidário que pode ajudar a salvar muitas vidas [...]" (E26). "Doar sangue é literalmente poder salvar a vida de alguém” (E27).

Nos relatos dos participantes, é notório que a prática da doação voluntária de sangue é vista como uma atitude solidária e que reflete o amor com o próximo, e é muito importante pela questão de estar salvando vidas através deste ato que se caracteriza como voluntário, altruísta e simples, já que nas doações espontâneas, normalmente o doador não conhece o 
receptor do sangue doado. Esses achados vão de encontro ao estudo de Coelho e Faria, (2018), ao apontarem que os participantes entrevistados verbalizaram que a doação voluntária de sangue é de suma importância, pois vidas poderão ser salvas mediante esta prática simples e não remunerada, sendo essencial para manutenção da vida.

Resultados semelhantes foram apontados no estudo de Brasil, Vargas, Vaz, Silva e Oliveira (2020), ao evidenciarem que os participantes entrevistados verbalizaram que a doação espontânea simboliza um ato solidário e humanístico, visto que se sentem satisfeitos em poder ajudar alguém que esteja no momento precisando de sangue para que possa se manter vivo, sem nem mesmo conhecer quem receberá o sangue doado (receptor). Gil, Cacciari, Cazañas e Maia (2018), pontuaram que a solidariedade e o amor ao próximo se configuram como características essenciais do processo de doação de sangue e se constituem nos principais elementos motivacionais para a realização desta ação, bem como para a fidelização de doadores no momento da primeira doação, tornando-se doadores habituais.

Em diversos países, incluindo o Brasil, a situação é preocupante em relação a doação de sangue e o número de doadores, já que a obtenção de sangue e/ou recrutamento de doadores voluntários é desproporcional ao percentual desejado. Mesmo diante de uma ampla divulgação por meio das estratégias de captação sobre a importância da doação espontânea, a realização desta prática ainda se torna cada vez mais baixa, colocando em risco a saúde e a vida daqueles que venham futuramente necessitar de transfusões sanguíneas (Bousquet; Aleluia \& Luz, 2018; Carlesso et al., 2017).

Diante dos achados, torna-se necessário cada vez mais, o recrutamento de doadores voluntários de sangue, uma vez que o único meio de obtenção de sangue é mediante a doação voluntária e altruísta, entretanto, nesta investigação se constatou que aproximadamente 63\% (n=17) dos participantes não são doadores de sangue. Diante deste dado, pode-se inferir que os estudantes da saúde desta pesquisa, mesmo conhecendo e expondo informações consistentes sobre a importância da doação de sangue, não realizam esta prática. Sobre o assunto, Otéro et al. (2020) trazem que o simples fato dos futuros profissionais de saúde não serem doadores, é uma questão preocupante, já que futuramente serão não apenas profissionais e sim educadores em saúde, onde serão responsáveis por informar, orientar e sensibilizar a população a se tornar doadora voluntária habitual. Logo, sob a ótica dos autores, destaca-se a importância dos universitários, primeiramente, se tornarem doadores de sangue.

Assim, esses resultados nos levam a admitir a necessidade de conhecer as razões pelas quais muitos estudantes não realizam este ato solidário, visto que o público jovem, conforme os apontamentos de Otéro et al. (2020), constitui um grupo ideal para realização das doações, pois geralmente apresentam um bom estado de saúde e tem muitos anos de vida pela frente para doar. Amorim e Baldessar (2019) acrescentam que desde 1980, a doação voluntária de sangue foi reconhecida, mundialmente, como um gesto voluntário, solidário e altruísta, sendo extremamente importante para a manutenção da vida, bem como para o funcionamento dos hemocentros e/ou hospitais, sendo que apenas por intermédio da doação de sangue que se é possível obter essa substância, assim fazendo-se necessária a procura constante de candidatos aptos à doação.

\section{Categoria 2: Necessidade da doação de sangue em situações envolvendo acidentes, doenças e cirurgias.}

Nesta categoria, verificou-se que a doação de sangue foi descrita pelos acadêmicos como uma prática de extrema importância em situações que envolvem acidentes, cirurgias, doenças, dentre outros eventos, posto que normalmente estas ocorrências, principalmente, as emergenciais, como é o caso dos acidentes e cirurgias, acontecem devido a uma perda considerável de sangue, conforme se evidencia nas falas a seguir:

"Extrema, tendo em vista a grande necessidade que se tem decorrente de acidentes e doenças" (E08). "Para ajudar os pacientes quando necessitarem de uma transfusão, ou quando perderem sangue em um acidente, durante uma cirurgia" (E11). "Ajudar as pessoas que precisam e que estejam passando por alguma enfermidade, a ter uma vida mais prolongada" (E14). "A doação de sangue é de extrema importância para pessoas necessitadas em ocasiões de 
cirurgia ou de alguma ocasião em que necessite de sangue" (E19). "Ajudar uma pessoa que precisa por motivos acidentais ou doenças" (E22). "Muitas pessoas necessitam do sangue doado, seja por uma doença, seja porque precisou na hora de uma cirurgia, seja por qualquer outro [...]” (E24).

Os depoimentos dos entrevistados evidenciaram que a doação voluntária de sangue é uma prática capaz de ajudar os indivíduos nas seguintes situações: acidentes, cirurgias, doenças, dentre outras ocasiões que carecem de transfusões sanguíneas. Dados similares foram evidenciados em uma pesquisa realizada em um dos hospitais universitários localizado no Rio de Janeiro, ao pontuarem que a doação espontânea de sangue é uma prática de suma importância, sendo muito utilizada em ocasiões envolvendo acidentes graves que, por ventura, são frequentes nos finais de ano; doenças hematológicas; procedimentos médicos (cirurgias e transplantes) e qualquer outra condição crítica que se faz necessário de transfusão (Silva \& Valadares, 2015).

Isso se justifica, em virtude de que as situações emergenciais, como é o caso de acidentes e cirurgias complexas, geralmente, ocorrem uma grande perda de sangue, o que poderá comprometer a saúde e a vida dos pacientes, consequentemente, é necessária a realização das doações para que possam manter o estoque de bolsas de sangue e assim suprir e acompanhar as demandas por transfusões sanguíneas (Marçal \& Dombrowski, 2017). Tais achados também foram apontados no estudo de Coelho e Faria (2018), ao evidenciarem que o ato voluntário de doar sangue é de suma importância, pois, caracteriza-se como um remédio vital que, poderá salvar a vida dos indivíduos, principalmente em ocasiões onde a demanda de transfusão é elevada, como nas situações envolvendo acidentes, cirurgias, morbidades, transplantes e dentre outras ocasiões.

A acadêmica E14 descreve a necessidade de doação de sangue para as pessoas que apresentam alguma enfermidade, cuja a finalidade da doação é o restabelecimento da saúde destes, para obter uma vida mais prolongada. Essa mesma percepção está no estudo de Forster et al. (2018), ao pontuarem que os pacientes que apresentam alguma enfermidade e/ou distúrbios hematológicos tendem a apresentar melhoras em seu quadro clínico e uma vida mais prolongada após receberem quantidade significativa de sangue.

Frente aos achados, nota-se que as percepções descritas pelos acadêmicos, corroboram com a literatura ao confirmar que o tecido sanguíneo é um elemento essencial nas situações mencionadas. Diante disso, pode-se inferir que os futuros profissionais deste estudo possuem conhecimento sobre a importância da doação voluntária de sangue, e a instituição de ensino superior, a qual eles pertencem, poderá desempenhar um papel social relevante no processo de doação de sangue, pois proporcionará a disseminação de informações acerca deste gesto solidário, seja por meio de ações educativas em saúde, projetos de extensão, rodas de conversas, pesquisas e feiras científicas, palestras, dentre outros. Além de que poderá sediar um ponto de coleta de sangue, facilitando esta ação e o recrutamento de candidatos elegíveis (Bousquet; Aleluia \& Luz, 2018).

\section{Considerações Finais}

Os universitários deste estudo, demonstraram que conhecem as situações em que a doação de sangue se faz necessária, descrevendo-a como um ato solidário e voluntário, que permite que vidas sejam salvas, considerando-a de extrema importância para a manutenção dos serviços de saúde, apesar da grande maioria desses estudantes não serem doadores voluntários de sangue. Desse modo, este estudo comprova a necessidade de se desenvolver ações de captação de doadores dentro das instituições de ensino superior, com o objetivo de difundir a importância desses futuros profissionais de saúde se tornarem doadores voluntários. Logo, ressalta-se como limitação do estudo, o tamanho amostral e esse ter sido realizado em apenas uma universidade pública da região centro-sul cearense.

Desta maneira, os resultados da pesquisa servirão de referência para o desenvolvimento de novos estudos, com outros delineamentos metodológicos. Recomenda-se assim, a realização de pesquisas mais amplas acerca da temática, envolvendo 
outros cenários, como por exemplo, as universidades privadas, de modo a analisar a realidade desses estudantes e sua percepção sobre o assunto, bem como, estudos que venham elucidar as razões pelas quais muitos dos universitários da área da saúde não são doadores de sangue, com o intuito de incidir sobre essas justificativas, traçando estratégias de captação de candidatos elegíveis à doação nessas instituições de ensino superior, para que assim, mudanças possam ser incorporadas ao meio acadêmico e campanhas possam ser planejadas, visando incentivar a realização da doação de sangue.

\section{Financiamento}

Este estudo contou com bolsista financiado pelo Programa Institucional de Bolsas de Iniciação Científica - PIBIC da Universidade Regional do Cariri (URCA) em parceria com o Fundo Estadual de Combate à Pobreza (FECOP).

\section{Referências}

Amorim, B. M., \& Baldessar, M. Z. (2019). Aspectos da doação de sangue entre acadêmicos de Medicina. Revista da AMRIGS, 63 (3), $273-278$.

Baldin, N., \& Munhoz, E. M. B. (2011). Educação Ambiental Comunitária: uma experiência com a técnica de pesquisa snowball (bola de neve). Rev. eletrônica Mestr. Educ. Ambient, 27,46-60.

Bardin, L. (2016). Análise de conteúdo. Edições 70.

Bousquet, H. M., Aleluia, I. R. C., \& Luz, L. A. (2018). Fatores decisivos e estratégias para captação de doadores em hemocen tros: revisão da literatura. Rev. Ciênc. Méd. Biol, 17(1), 84-88.

Brasil. (2015). Ministério da Saúde. Secretaria de Atenção à Saúde. Departamento de Atenção Especializada e Temática. Manual de Orientações para Promoção da Doação Voluntária de Sangue. Brasília, DF: Ministério da Saúde.

Carlesso, L., Guimarães, R. F. S., Silva, S. L., Santos, C. F., Viero, V., Vieira, S. V., \& Perlini, N. M. O. G. (2017). Estratégias Implementadas em Hemocentros Ppara Aumento da Doação de Sangue. Rev Bras Promoç Saúde, 30(2), 213-220.

Coelho, C., \& Faria, M. D. (2018). Intenções Podem Salvar Vidas? Motivações e Dificuldades de Potenciais Doadores de Sangue À Luz do Marketing Social. Ciências Sociais em Perspectiva, 17(33), 157-178.

Faleiros, F., Käppler, C., Pontes, F. A. R., Silva, S. S. C., Goes, F. S. N., \& Cucick, C, D. (2016). Uso de Questionário Online e Divulgação Virtual como Estratégia de Coleta de Dados em Estudos Científicos. Texto Contexto Enferm, 25(4) e3880014, 1-6.

Forster, F., Câmara, A. L., Moraes, C. L. K, Honório, M. T., Mattia, D., \& Lazzari, D. D. (2018). Percepção Dos Enfermeiros Quanto à Assistência de Enfermagem no Processo Transfusional. Enferm. Foco, 9 (3): 71-75.

Gil, M. D., Cacciari, P., Cazañas, E. F. \& Maia, M. R. G. (2018). Contribuições de Atividades Educativas Realizadas na Sala de Espera para o Acadêmico de Enfermagem. Revista de Enfermagem do Centro-Oeste Mineiro, 8(e2103), 1-8.

Locks, M. O. H., Salum, N. C., Barros, B. S., Matos, E., Anders, J. C., \& Schneider, D. G. (2019). Perfil dos doadores de san gue que apresentaram reações adversas à doação. Rev Bras Enferm, 72(1), 87-94.

Malheiros, G. C., Oliveira, A. A. S. T., Pinheiro, C. B., Monteiro, K. N. O., \& Abreu, A. M. O. W. (2014). Fatores associados à motivação da doação sanguínea. Revista Científica da FMC, 9(1), 8-12.

Marçal, M. P., \& Dombrowski, P. A. (2017). Percepção dos Acadêmicos de Enfermagem quanto aos Cuidados na Transfusão de Hemocomponentes. Publ. UEPG Ci. Biol. Saúde, 23 (2), 150-158.

Minayo M.C.S. (2017). Sampling and saturation in qualitative research: consensuses and controversies. Rev Pesq Qual. 7(5):1-12.

Nascimento, A. A., Ilha, S., Marzari, C. K., Diefenbach, G. D., \& Backes, D. S. (2015). Cuidado de Enfermagem no Processo de Doação de Sangue: Percepção dos Profissionais e dos Doadores. R. Enferm. Cent. O. Min, 5(1), 1497-1504.

Nascimento L. C. N., Souza T. V., Oliveira I. C. S., Moraes J. R. M. M., Aguiar R. C. B., Silva, L. F. (2018). Theoretical saturation in qualitative research: an experience report in interview with schoolchildren. Rev Bras Enferm. 71(1):243-8.

Ótero, L. C., Marques, E., Santos, A. E. M., González, R. R., \& Iglesia, J. D. C. F. (2020). Conhecimento de estudantes portugueses de enfermagem sobre doação de sangue. Acta Paul Enferm, 33(eAPE20190166), 1-7.

Pereira, J. R., Sousa, C. V., Matos, B. E., Rezende, L. B. O., Bueno, N. X., \& Dias, A. M. (2016). Doar ou não doar, eis a questão: uma análise dos fatores críticos da doação de sangue. Cien Saude Colet. 21(8), 2475-484.

Raghuwanshi, B., Pehlajani, N. K., \& Sinha, M. K. (2016). Voluntary Blood Donation among Students - A Cross-Sectional Study on Knowledge and Practice vs. Attitude. J Clin Diagn Res, 10 (10), 18-22. 
Research, Society and Development, v. 10, n. 5, e19710514887, 2021 (CC BY 4.0) | ISSN 2525-3409 | DOI: http://dx.doi.org/10.33448/rsd-v10i5.14887

Silva, G. E. M., \& Valadares, G. V. (2015). onhecendo os Meandros da Doação de Sangue: Implicações para a Atuação do En fermeiro na Hemoterapia. Rev Bras Enferm, 68(1), 32- 9.

Smeltzer, S. C., Bare, B.G., Hinkle, J. L \& Cheever,K. H. (2014). Brunner \&amp; Suddarth, Tratado de Enfermagem médico cirúrgica. Guanabara Koogan.

Souza, M. K. B., \& Santoro, P. (2019). Desafios e estratégias para doação de sangue e autossuficiência sob perspectivas regionais da Espanha e do Brasil. Cad. Saúde Colet, 27 (2), 195-201.

Vinuto, J. A. (2014). A amostragem em Bola de Neve na Pesquisa Qualitativa: Um Debate em Aberto. Temáticas, 22 (44), 203-220. 